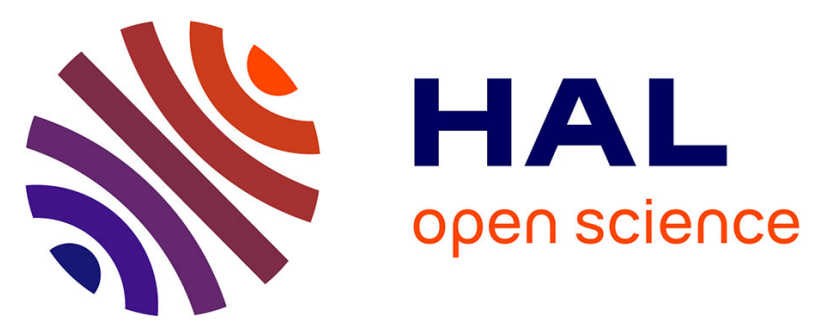

\title{
AFM study of hydrodynamics in boundary layers around micro- and nanofibers
}

Julien Dupré de Baubigny, Michael Benzaquen, Caroline Mortagne, Clémence

Devailly, Sébastien Kosgodagan Acharige, Justine Laurent, Audrey

Steinberger, Jean-Paul Salvetat, Jean-Pierre Aimé, Thierry Ondarçuhu

\section{To cite this version:}

Julien Dupré de Baubigny, Michael Benzaquen, Caroline Mortagne, Clémence Devailly, Sébastien Kosgodagan Acharige, et al.. AFM study of hydrodynamics in boundary layers around micro- and nanofibers. Physical Review Fluids, 2016, 1, pp.44104 - 44104. 10.1103/PhysRevFluids.1.044104. ensl-01474528

\section{HAL Id: ensl-01474528 \\ https://hal-ens-lyon.archives-ouvertes.fr/ensl-01474528}

Submitted on 22 Feb 2017

HAL is a multi-disciplinary open access archive for the deposit and dissemination of scientific research documents, whether they are published or not. The documents may come from teaching and research institutions in France or abroad, or from public or private research centers.
L'archive ouverte pluridisciplinaire HAL, est destinée au dépôt et à la diffusion de documents scientifiques de niveau recherche, publiés ou non, émanant des établissements d'enseignement et de recherche français ou étrangers, des laboratoires publics ou privés. 


\title{
AFM study of hydrodynamics in boundary layers around micro- and nanofibers
}

\author{
Julien Dupré de Baubigny, ${ }^{1,2}$ Michael Benzaquen, ${ }^{3,}{ }^{*}$ Caroline Mortagne, ${ }^{1,2}$ Clémence Devailly, ${ }^{4}$ \\ Sébastien Kosgodagan Acharige, ${ }^{4}$ Justine Laurent, ${ }^{4}$ Audrey Steinberger, ${ }^{4}$ Jean-Paul Salvetat ${ }^{5}$ \\ Jean-Pierre Aimé, ${ }^{6}$ and Thierry Ondarçuhu ${ }^{1, \dagger}$ \\ ${ }^{1}$ Nanosciences Group, CEMES, CNRS, UPR 8011, 29 rue Jeanne Marvig, \\ 31055 Toulouse cedex 4, France \\ ${ }^{2}$ Université de Toulouse, 29 rue Jeanne Marvig, 31055 Toulouse cedex 4, France \\ ${ }^{3}$ Laboratoire de Physico-Chimie Théorique, CNRS, UMR 7083 Gulliver, ESPCI ParisTech, \\ PSL Research University, 10 rue Vauquelin, 75231 Paris cedex 5, France \\ ${ }^{4}$ Laboratoire de Physique, Université de Lyon, Ens de Lyon, Université Claude Bernard Lyon 1, \\ CNRS, 69342 Lyon, France \\ ${ }^{5}$ CRPP, CNRS, UPR 8641, 115 avenue du Dr. Albert Schweitzer, 33600 Pessac, France \\ ${ }^{6}$ CBMN, CNRS, UMR 5248, 2 rue Escarpit, 33600 Pessac, France
}

(Received 29 February 2016; published 22 August 2016)

\begin{abstract}
The description of hydrodynamic interactions between a particle and the surrounding liquid, down to the nanometer scale, is of primary importance since confined liquids are ubiquitous in many natural and technological situations. In this paper we combine three nonconventional atomic force microscopes to study hydrodynamics around microand nanocylinders. These complementary methods allow the independent measurement of the added mass and friction terms over a large range of probe sizes, fluid viscosities, and solicitation conditions. A theoretical model based on an analytical description of the velocity field around the probe shows that the friction force depends on a unique parameter, the ratio of the probe radius to the thickness of the viscous boundary layer. We demonstrate that the whole range of experimental data can be gathered in a master curve, which is well reproduced by the model. This validates the use of these atomic force microscopy modes for a quantitative study of hydrodynamics and opens the way to the investigation of other sources of dissipation in simple and complex fluids down to the submicron scale.
\end{abstract}

DOI: 10.1103/PhysRevFluids.1.044104

\section{INTRODUCTION}

The design of multiscale functional networks with microfluidic channels produces a wealth of new experiments and concepts in which the attempt to understand and control the flow of heterogeneous fluids bearing microparticles and nanoparticles is of primary interest. There are now many possible ways to study properties at a microscopic level [1], giving new insight into phenomena that often exhibit features at the macroscopic scale. Applications are numerous in many transversal domains of major interest where the behavior of confined fluid is of primary importance. Within this framework, determining the relevant lengths and scaling laws that govern hydrodynamic interactions is a major goal. The flow around particles is essential to interpret dynamic light scattering experiments [2] or to understand the rheological properties of colloidal dispersions [3]. In particular, the transport properties of rodlike particles [2] have known a renewed interest due to the development of carbon nanotubes suspensions [4]. The control of flow inside channels is also of primary importance for the further development of micro- and nanofluidics. Indeed, many digital fluidic networks are elaborated for screening purposes where spatially localized chemical reactions are planned to operate as a

\footnotetext{
*Present address: Capital Fund Management, 23 rue de l'Université, 75007 Paris, France.

†ondar@cemes.fr
} 
hierarchically organized set of logical gate functions [5]. In the case of flow of suspensions confined inside microchannels, the hydrodynamic interactions mediated by the embedding liquid lead to anomalous diffusion of the particles [6]. Confined complex fluids are also ubiquitous in life sciences since many biological processes involve biofluids inside vascular systems [7] or, at a smaller scale, in aquaporin [8]. A fine understanding of the microscopic hydrodynamic coupling between particles is also crucial for the controlled collective motion [9-11] of assemblies of motile particles such as micro- or nanoswimmers [12]. The hydrodynamic interaction can also be used to manipulate nanoobjects as, for example, the flow-induced structuring of colloidal suspensions [13] or the translocation and stretching of polymers in nanochannels for ultrafiltration [14]. The stress resulting from the fluid velocity field gradient at the wall can even induce the scission of nanotubes under sonication [15].

In all these systems one has to carefully manage the boundary constraints that determine the fluid mechanical properties and flow behavior. This includes the issue of slip at the solid wall, which has been intensely debated [16], but geometry and size effects of the system, whether a channel or a particle, also matter.

In the present work we use three different atomic force microscopy (AFM) modes to extract the conservative and dissipative contributions of a small volume of fluid surrounding an oscillating nano-object. The aim is to quantify to what extent the surrounding fluid is perturbed by a nanoparticle motion. A quantitative knowledge of the fluid contribution, as well as of the extension of the velocity field upon the action of a unique moving nano-object, first provides information on the energy one has to supply to ensure a stationary state and second gives a length that determines the range of the hydrodynamic interactions.

Few techniques are available to probe locally the flow behavior around probes with micro- or nanometer scale. Microrheology techniques have been developed to address some of the abovementioned issues $[17,18]$. They usually rely on the monitoring of the Brownian motion of micronsized beads or on the measurement of their interaction with the fluid when manipulated by optical tweezers [19]. The results of these passive or active methods are related through the fluctuationdissipation theorem. This can be downscaled by monitoring the dynamic response of magnetic nanoparticles to an oscillating magnetic field [20]. Several miniaturized sensors based on micromechanical devices [21] or piezoelastic vibrators [22] have also been developed to measure the viscosity down to dimensions of the order of $10 \mu \mathrm{m}$. The mechanical response of microfabricated cantilevers immersed in the fluid under study has also been used but is limited to gases [23] or liquids of low viscosities $(<10 \mathrm{mPas})$ because of the strong damping of the cantilever oscillation [24,25]. Interaction with polymer layers has also been studied by such techniques [26]. An alternative is to use a cantilever with a hanging fiber partially dipped in the fluid [27-29]. The advantage is that the cantilever itself is not damped by the liquid, allowing a precise measurement of the interaction of the fiber with the liquid. Quartz microbalances can also be used to probe liquids at interfaces at MHz frequencies [30], while pump-probe optical spectroscopy methods provide a way to reach $\mathrm{GHz}$ frequencies [31].

In this paper we use the hanging-fiber geometry (Fig. 1) with three different AFM setups: (i) a commercial AFM setup operated in the frequency-modulation (FM) mode giving the response of the liquid to an oscillatory excitation in the $50-500 \mathrm{kHz}$ range, (ii) a recently developed AFM based on a microelectromechanical system (MEMS) working at high frequency around 10-50 MHz [32,33], and (iii) a homemade high-resolution (HR) AFM [34] used to measure the influence of the liquid interaction on the thermal fluctuations of the cantilever [27,28]. All methods allow the independent monitoring of the conservative and dissipative contributions of the interaction with the tip. In order to reach precise quantitative information we chose a simple geometry consisting in probes with a micro- or nanocylindrical fiber tailored at the extremity of an AFM cantilever.

In the following we show that this geometry associated with nonconventional AFM setups allows for quantitative study of the hydrodynamic interaction of a micro- or nanosized probe with liquids. A hydrodynamic model is proposed to interpret the whole set of data covering a large range of solicitations and experimental conditions (probe size and liquid properties) to give a unified picture of the phenomena at stake. 


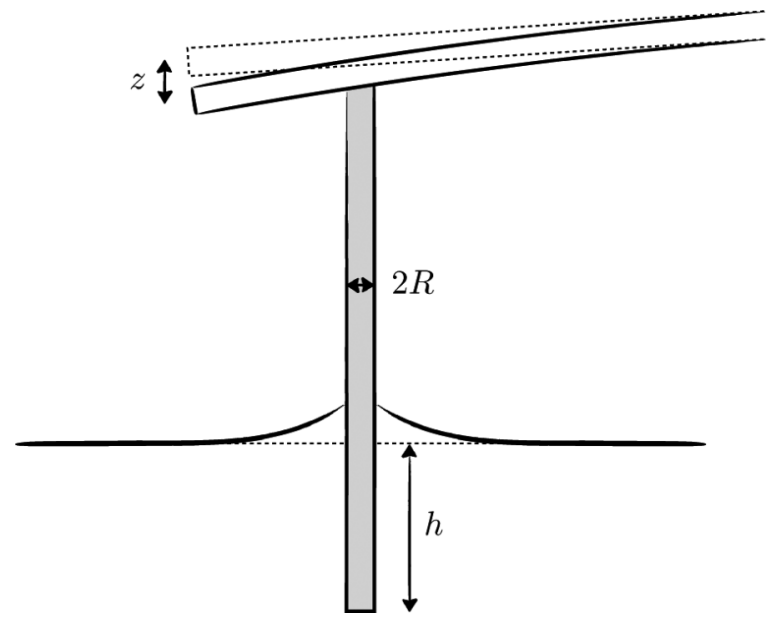

FIG. 1. Schematic representation of the experiment. The extremity of a fiber with radius $R$ is dipped over a height $h$ with respect to the reference level of the liquid interface while monitoring the $z$ deflection of the cantilever.

In Sec. II we describe the experimental conditions, the different instruments and operating AFM modes, and the raw data that are monitored during the immersion of the probe in the liquid. In Sec. III we show how physical quantities can be extracted from the raw data. In Sec. IV a theoretical model is presented and compared to the experimental results.

\section{EXPERIMENTAL METHODS AND RAW DATA}

The tips were dipped in a container drilled in an aluminum or copper sample holder filled with the liquid under study or in a droplet supported by a silicon substrate, both with diameter $\geqslant 5 \mathrm{~mm}$ and depth $\geqslant 1 \mathrm{~mm}$. We used a large series of liquids including alkanes, long-chain alcohols, glycols, silicon oils, and ionic liquids. These liquids were chosen to cover a large range of viscosities (from 1 to $1000 \mathrm{mPa}$ s), but they also differ by their surface tension and consequently by the contact angle on the fibers. The chemical nature of the liquid may also come into play, in particular for ionic liquids which lead to strong structuration at solid interfaces [35]. The relevant parameters (volumic mass $\rho$ and viscosity $\eta$ ) of the liquids used are listed in the Supplemental Material [36]. The experiments were performed by using three complementary AFM setups operated in two different modes with micro- and nanosized probes.

\section{A. Frequency-modulation FM AFM measurements}

The first series of measurements were performed on a PicoForce AFM (Bruker) operated in the FM AFM mode using a phase-locked loop device (HF2PLL, Zurich Instruments). In this mode, the cantilever oscillates at one of its resonance frequencies (fundamental mode $f_{0}$ or second mode $\left.f_{1}\right)$ and the frequency shift $\Delta f$ compared to the oscillation in air is monitored. A proportionalintegral-differential closed loop was used to modulate the excitation signal $A_{e x}$ (in volts) sent to the piezoelectric element in order to maintain the amplitude of oscillation of the tip constant. The monitoring of the $A_{e x}$ signal gives access to the dissipation of the system. The advantage of the FM AFM mode compared to the standard amplitude modulation (AM AFM) mode is that it allows measuring independently the conservative and dissipative parts of the interaction while maintaining the oscillation amplitude constant.

We used two types of AFM tips terminated by a nanocylinder with diameter below $60 \mathrm{~nm}$. These tips are made by focused ion beam milling of a silicon tip (CDP55 by Team Nanotec, Germany) or by growth of an $\mathrm{Ag}_{2} \mathrm{Ga}$ nanoneedle at the tip extremity (Nauga Needles, USA). Both types of 


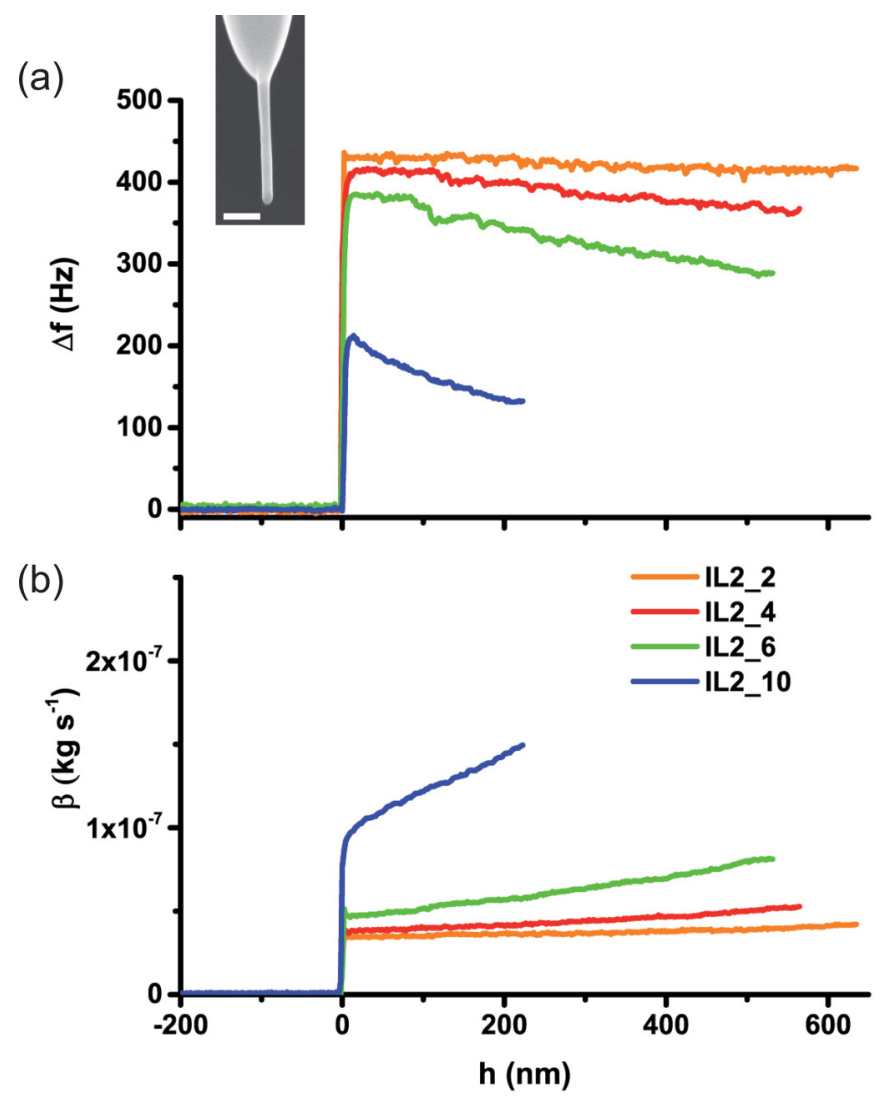

FIG. 2. (a) Frequency shift and (b) friction coefficient $\beta$ as a function of the immersion depth $h$ for a series of four ionic liquids with viscosities of $36.5,110,200$, and $500 \mathrm{mPa}$ s from IL2_2 to IL2_10. The inset shows a SEM image of the tip used with a diameter of $55 \mathrm{~nm}$ and length $680 \mathrm{~nm}$; the scale bar is $200 \mathrm{~nm}$.

tips were mounted on cantilevers with a static spring constant of the order of $2 \mathrm{~N} / \mathrm{m}$, soft enough to perform static deflection measurements while being adapted for dynamic AFM studies. Quality factors $Q$ were of the order of 200-300. Measurements were performed both on the fundamental mode with a resonance frequency of the order of $f_{0} \sim 70 \mathrm{kHz}$ and on the second mode with a resonance frequency $f_{1}=6.25 f_{0} \sim 440 \mathrm{kHz}$. The associated spring constants, measured using the thermal noise spectrum, are $k_{0} \sim 2 \mathrm{~N} / \mathrm{m}$ and $k_{1}=40 k_{0} \sim 80 \mathrm{~N} / \mathrm{m}$ [37].

The results of a typical experiment are plotted in Fig. 2. They are obtained with a silicon tip ended by a nanocylinder with radius $27.5 \mathrm{~nm}$ and length $680 \mathrm{~nm}$ (see the inset in Fig. 2) oscillating at its resonance frequency ( $f_{0}=72450 \mathrm{~Hz}$ in air) with an amplitude of $7 \mathrm{~nm}$. The tip was dipped in and withdrawn from the liquid bath with a ramp amplitude of $1 \mu \mathrm{m}$ and a velocity of $2 \mu \mathrm{m} / \mathrm{s}$. The frequency shift $\Delta f$ and friction coefficient $\beta$ (deduced from $A_{e x}$ as explained below) are reported as a function of the immersion depth $h$ for one series of ionic liquids.

The following three different signals were monitored during this process:

(i) The deflection of the cantilever measures the capillary force $F_{\text {cap }}$, which gives information about the wetting properties of the nanocylinder. Note that buoyancy forces $F_{b}$ are negligible in the range of probe sizes used $\left(\frac{F_{b}}{F_{\text {cap }}}<10^{-3}\right)$. Since in this paper we do not consider the effects of the meniscus close to the contact line, this curve is not shown. Two plateaus are observed when the tip is dipped in and then withdrawn from the liquid bath, corresponding to the advancing and receding contact angles as already discussed in several papers [38-40]. 
(ii) The frequency shift $\Delta f$ compared to the oscillation in air [Fig. 2(a)] exhibits a large positive jump when the meniscus is formed. When the tip is dipped further into the liquid a linear decrease of the resonance frequency is observed with a slope all the stronger that the liquid is viscous. Since the frequency of the cantilever is given by $f=\frac{1}{2 \pi} \sqrt{\frac{k_{c}}{m_{c}}}$, where $k_{c}$ and $m_{c}$ are, respectively, the spring constant and the effective mass of the cantilever, the frequency shift may have two origins: a change of the spring constant $\Delta k$ or a change of mass $\Delta m$ according to $\frac{\Delta f}{f}=\frac{1}{2} \frac{\Delta k}{k_{c}}-\frac{1}{2} \frac{\Delta m}{m_{c}}$. The positive jump of $\Delta f$ observed at the meniscus formation can be attributed to the meniscus effective spring constant $k_{\text {men }}$ [41], which depends on the geometrical shape of the meniscus related to the surface tension and the contact angle [39,41], whereas the decrease may be a mass effect due to the liquid around the fiber.

(iii) The normalized excitation $\frac{A_{e x}-A_{0}}{A_{0}}$ gives the relative change in excitation $A_{e x}$ required to maintain the amplitude constant compared to the situation in air $A_{0}$ [Fig. 2(b)]. In order to obtain quantitative information, the dissipation is characterized by the friction coefficient $\beta=\beta_{0}\left(\frac{A_{e x}-A_{0}}{A_{0}}\right)$, where $\beta_{0}=\frac{k_{c}}{\omega Q}$ is the friction coefficient in air far from the surface [42] with $\omega=2 \pi f$ the angular frequency and $Q$ the quality factor of the cantilever. A jump of dissipation in the $\beta(h)$ curve is observed when the meniscus is formed and a linear increase with the immersion depth $h$ is obtained when dipping the fiber further into the liquid. Again, this slope increases significantly with the liquid viscosity. The same signals can also be recorded on the very same system using the second mode of the cantilever of frequency $f_{1}=455400 \mathrm{~Hz}$, which allows assessing the influence of excitation rate.

\section{B. High-frequency MEMS AFM measurements}

The microelectromechanical resonators used for the high-frequency measurements were designed and fabricated by Walter and co-workers at IEMN (Lille, France). Details are reported elsewhere [32,33]. The resonating element is a ring anchored by four points located at vibration nodes [Fig. 3(a)] and is equipped with a sharp pyramidal tip of $5^{\circ}$ half angle [Fig. 3(b)]. The MEMS device was integrated in a specifically designed homemade AFM microscope (see Supplemental Material [36] for further details on the setup). The periodic forcing of the resonator and signal acquisition from the microwave detection circuit were performed with a lock-in amplifier including a phase-locked loop (HF2LI-PLL from Zurich Instrument). Since the MEMS AFM was operated in frequency modulation mode, we monitored the same signals, frequency shift $\Delta f$, and excitation amplitude $A_{e x}$ as for the FM AFM described in the previous section.

The results of a typical experiment are plotted in Figs. 3(c) and 3(d). They were obtained with a silicon tip with a pyramidal tip oscillating at its resonance frequency $\left(f_{0}=13.1 \mathrm{MHz}\right.$ in air) with an amplitude of $1 \mathrm{~nm}$. The quality factor of the oscillator is of the order of 700. The tip was dipped in and withdrawn from the liquid droplet with a ramp amplitude of $3 \mu \mathrm{m}$ and a velocity of $0.3 \mu \mathrm{m} / \mathrm{s}$. The graphs of frequency shift $\Delta f$ [Fig. 3(c)] and friction $\beta$ (deduced from $A_{e x}$ ) [Fig. 3(d)] are reported as a function of the immersion depth $h$ for a series of ethyleneglycols.

At the meniscus formation, a small negative-frequency shift is observed followed by a monotonic decrease of the frequency shift upon further dipping of the tip. This latter point is similar to the negative slope observed by FM AFM. Note that, due to the large value of the resonator spring constant compared to the meniscus spring constant $\left(\frac{k_{\text {men }}}{k_{c}} \sim 10^{-7}\right)$, no positive jump is observed at $h=0$. The dissipation signal also follows similar trends as for FM AFM with a jump in dissipation at the contact with liquid and an increase for positive $h$ values. As discussed in the next section the nonlinear behavior can be attributed to the pyramidal shape of the tip.

\section{High-resolution HR AFM measurements}

The thermal noise spectrum of the deflection $z$ of the cantilever was recorded using a home-built high-resolution quadrature phase interferometer, which measures the optical path difference between a laser beam reflecting on the free end of the cantilever above the location of the fiber and a reference 
(a)

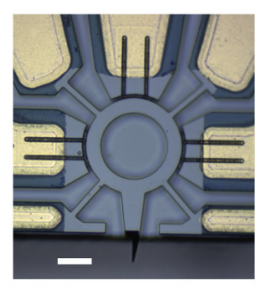

(b)

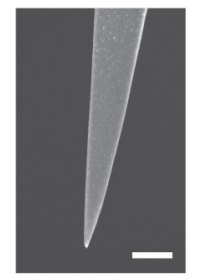

(c)

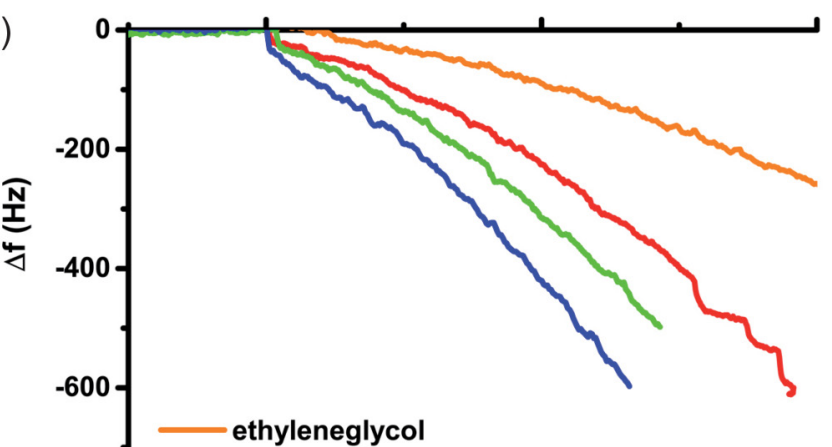

(d)

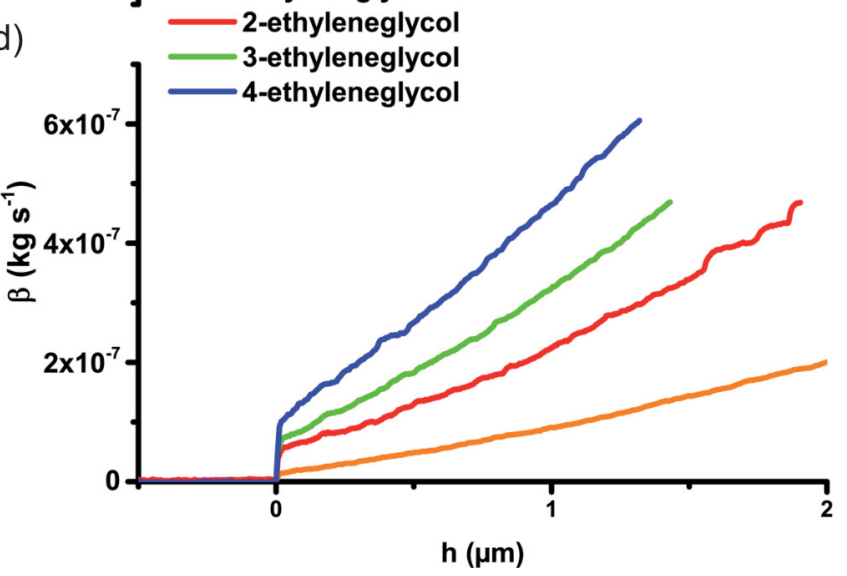

FIG. 3. (a) Optical micrograph of the resonating element; the scale bar is $20 \mu \mathrm{m}$. (b) SEM image of the tip extremity; the scale bar is $500 \mathrm{~nm}$. (c) Frequency shift $\Delta f$ as a function of the depth $h$ of immersion of the tip for a series of four polyethyleneglycols. (d) Same as (c) but for the friction coefficient $\beta$. The viscosities of the liquids used are 16, 37, 49 and $58 \mathrm{mPa}$ s from ethyleneglycol to tetraethylene glycol.

beam reflecting on the base of the cantilever [34]. This technique offers a very low detection noise [down to $10^{-14} \mathrm{~m} /(\mathrm{Hz})^{1 / 2}$ ] and is intrinsically calibrated against the laser wavelength. As probes we used elongated micrometer-sized glass cylinders glued on standard AFM cantilevers (Budget Sensors AIO, levers A and B) and cut between a sharp tweezers and a diamond tool, as described in Ref. [27]. These cylinders, typically 1-10 $\mu \mathrm{m}$ in diameter and 150-250 $\mu \mathrm{m}$ long are glued on a cantilever having a resonant frequency in air $f_{0} \sim 10 \mathrm{kHz}$ (spring constant $k_{0} \sim 0.25 \mathrm{~N} / \mathrm{m}$ ) and a quality factor of the order of $Q \sim 70$. The power spectral density (PSD) of the thermal fluctuations of the cantilever was measured as a function of the dipping depth $h$, which was varied by 3- or $5-\mu \mathrm{m}$ steps, allowing the system to relax prior to measurement [27]. For viscosities below or equal to $20 \mathrm{mPas}$, the experimental $P S D$ is fitted around the fundamental resonance peak using a model of simple harmonic oscillator. The data analysis gives access to the frequency shift $\Delta f$ and to the dissipation coefficient $\beta$ associated with the interaction of the tip with the liquid. For viscosities higher than $20 \mathrm{mPas}$, the thermal fluctuations of the cantilever are overdamped. The dissipation 


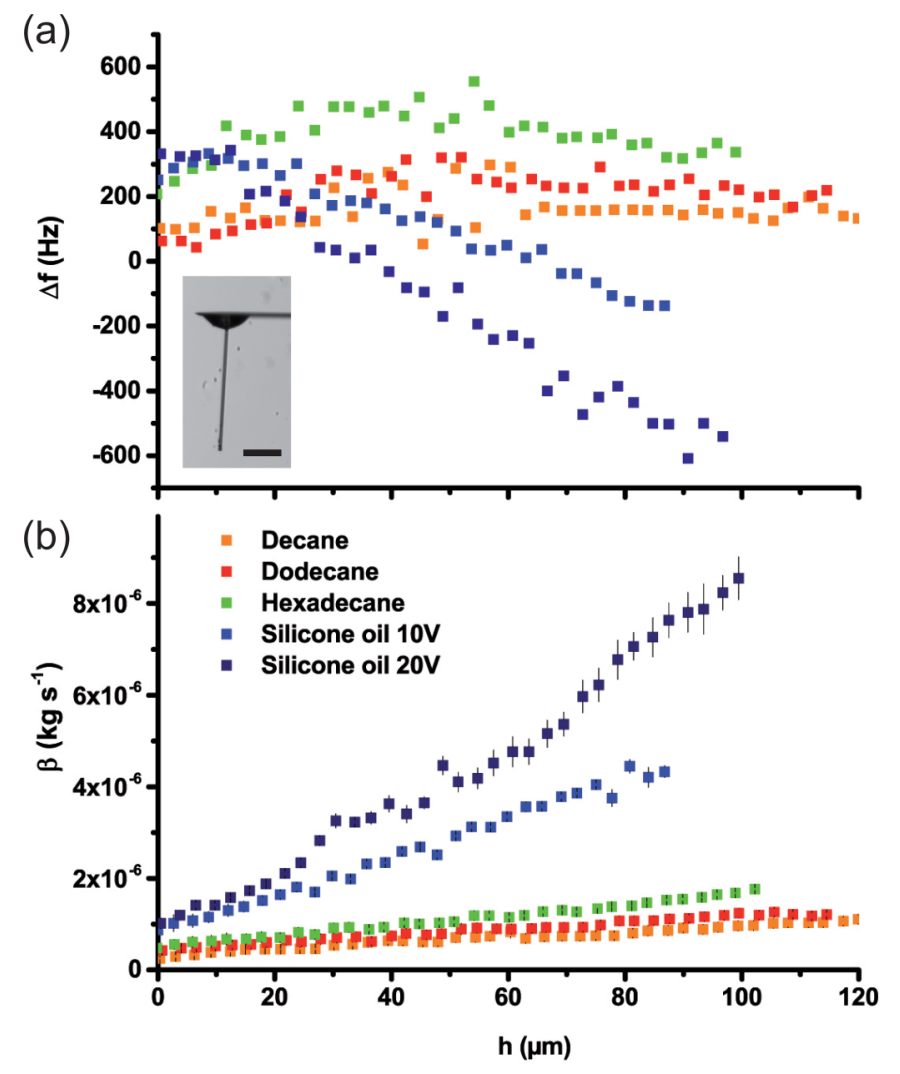

FIG. 4. (a) Frequency shift and (b) friction coefficient $\beta$ as a function of the immersion depth $h$ for five different liquids. The inset shows the optical micrograph of the glass fiber attached to an AFM cantilever; the scale bar is $50 \mu \mathrm{m}$. The viscosities of the liquids used are $0.85,1.36$, and $3.05 \mathrm{mPa}$ s from decane to hexadecane and 10 and $20 \mathrm{mPa}$ s for silicone oils of $10 \mathrm{~V}$ and $20 \mathrm{~V}$, respectively.

coefficient $\beta$ can still be obtained by fitting the experimental PSD by a Lorentzian around the cutoff frequency. However, no information can be obtained on the resonant frequency shift $\Delta f$ in this overdamped regime. More details on the data analysis procedure are given in the Supplemental Material [36], including a discussion about the related measurement uncertainty.

The frequency shift $\Delta f$ with respect to the resonant frequency $f_{0}$ in air is plotted as a function of the dipping depth $h$ for the same probe in several liquids in Fig. 4(a). A negative slope is clearly seen for dipping depths larger than $30 \mu \mathrm{m}$, that is to say, deep enough for the meniscus to be above the sharp defects created close to the cylinder's end by the cutting procedure (which could be responsible for a local change in the meniscus spring constant). The friction coefficient $\beta(z)$ plotted in Fig. 4(b) for the same series of liquids shows a positive slope. These behaviors are similar to the ones observed for FM AFM and MEMS AFM, the general trend being an increase of the slopes with the liquid viscosity.

The three AFM techniques therefore give access to similar quantities, namely, the frequency shift $\Delta f$ and the friction coefficient $\beta$ obtained in different but complementary conditions: The FM AFM and MEMS AFM use nanoprobes with radius in the $20-500 \mathrm{~nm}$ range with a variable forcing (1-100 $\mathrm{nm}$ for FM AFM and $0.5 \mathrm{pm}$ to $1 \mathrm{~nm}$ for MEMS AFM), whereas thermal noise is a passive method that applies better for micron-sized probes. FM AFM and MEMS AFM monitor in real time both frequency shift and excitation quantities and therefore the precise evolution of these quantities during the dipping process. On the contrary, thermal noise PSD can be recorded only at given heights $h$ but yields a complete noise spectrum response. Indeed, FM AFM and MEMS AFM are 
JULIEN DUPRÉ DE BAUBIGNY et al.

TABLE I. Range of parameters for the three AFM techniques.

\begin{tabular}{|c|c|c|c|c|c|c|}
\hline Technique & Forcing & Frequency & $\begin{array}{l}\text { Amplitude } \\
\text { range }\end{array}$ & $\begin{array}{c}\text { Probe radius } \\
\qquad R\end{array}$ & $\begin{array}{l}\text { Liquid viscosity } \\
\text { range }\end{array}$ & $\begin{array}{c}\text { Viscous layer } \\
\text { thickness } \delta\end{array}$ \\
\hline $\mathrm{FM}$ & 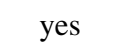 & $60-50$ & $1-1$ & $20-5$ & 10 & $5-5$ \\
\hline MEMS AFM & ye & $13 \mathrm{MHz}$ & $0.5 \mathrm{pm}$ to $1 \mathrm{~nm}$ & $100 \mathrm{~nm}$ to $1 \mu \mathrm{m}$ & $1-1000 \mathrm{mPa} \mathrm{s}$ & $0.1-1 \mu \mathrm{m}$ \\
\hline HR AFM & no & $2-100 \mathrm{kHz}$ & $\sim \AA$ & $500 \mathrm{~nm}$ to $5 \mu \mathrm{m}$ & $1-500 \mathrm{mPa} \mathrm{s}$ & $10-100 \mu \mathrm{m}$ \\
\hline
\end{tabular}

well adapted to small variations of quality factor $Q$ on narrow resonance peaks, whereas thermal noise is better adapted to strong dissipation leading to small- $Q$ values. The association of these techniques gives access to a large range of conditions in terms of solicitation, probe size, and liquid viscosity summarized in Table I. Interestingly, the behavior of the liquid can be probed over four decades of frequency and five decades of amplitude. The radius of the probe can also be changed over two orders of magnitude, whereas the different techniques have rather similar limitations in terms of measurable liquid viscosities. For FM AFM and MEMS AFM modes, we did not observe any influence of the oscillation amplitude in the range from $1 \mathrm{pm}$ to $20 \mathrm{~nm}$ on the measurements of $\Delta f$ and $\beta$, which indicates that all measurements were performed in the linear regime (see the Supplemental Material [36]).

\section{RESULTS: ADDED MASS AND DISSIPATION COEFFICIENT}

This article focuses on the behavior of the liquid around the immersed part of the fiber. With that aim, the choice of cylindrical tips is of primary importance: It ensures that as the tip is dipped into the liquid, the meniscus slips on the cylinder without a notable change of its geometry. As a consequence, the meniscus, like the end of the fiber, has a constant effect during the immersion or the withdrawal phase. Therefore, considering the slope of the experimental curves in their linear domain [ $h>0$ for the nanoneedles and $h>25 \mu \mathrm{m}$ for the glass microfiber (see Figs. 2-4)] unambiguously allows us to probe the influence of the liquid around the fiber.

In order to relate the raw data to physical quantities, we consider the drag force $F_{l}$ exerted by the surrounding liquid (fiber end excepted) on the fiber surface. Such force is generically written as the sum of a friction term and an added mass term [43], which qualitatively explains the shape of the aforementioned experimental curves.

(i) If the positive jump of the resonance frequency at the meniscus formation is induced by an additional stiffness to the system [41], the decrease observed for positive $h$ results from a mass effect according to $\frac{\Delta f}{f}=\underbrace{\frac{1}{2} \frac{k_{\text {men }}}{k_{c}}}_{\text {constant }}-\frac{1}{2} \frac{\Delta m}{m_{c}}$. The oscillating fiber drags a layer of liquid that increases with the immersed length. This corresponding added mass, per unit length, can be calculated using $m^{*}=-2 m_{c}\left(\frac{\Delta f}{f}\right)^{*}$, where $\left(\frac{\Delta f}{f}\right)^{*}$ is the relative frequency shift per unit length $h$.

(ii) Accordingly, the shape of the dissipation curves $\beta(h)$ can be interpreted by a jump due to the dissipation in the meniscus and at the end of the tip, followed by a positive slope resulting from the increase of the friction coefficient with the immersed length of the fiber. The friction coefficient per unit length $\beta^{*}$ is therefore directly measured as the slope of the friction coefficient $\beta$ for positive $h$.

Note that for MEMS AFM, the tips are not cylindrical but have the shape of a sharp pyramid (half angle $\alpha=5^{\circ}$ ). The linear increase of the radius with the immersion $h$ leads to an increase of the slopes with $h$. In a rough approximation, the volume of fluid involved with the oscillating motion scales as $\delta h^{2} \tan \alpha$, where $\delta$ is the thickness of the liquid layer dragged by the probe. In the following, we therefore measured the slope of the $m^{*}(h)$ and $\beta^{*}(h)$ curves, just after the meniscus formation $(h=0)$. The corresponding wetted radius $R$ was deduced from the meniscus height, which is obtained from the hysteresis between advancing and retracting curves [40]. 


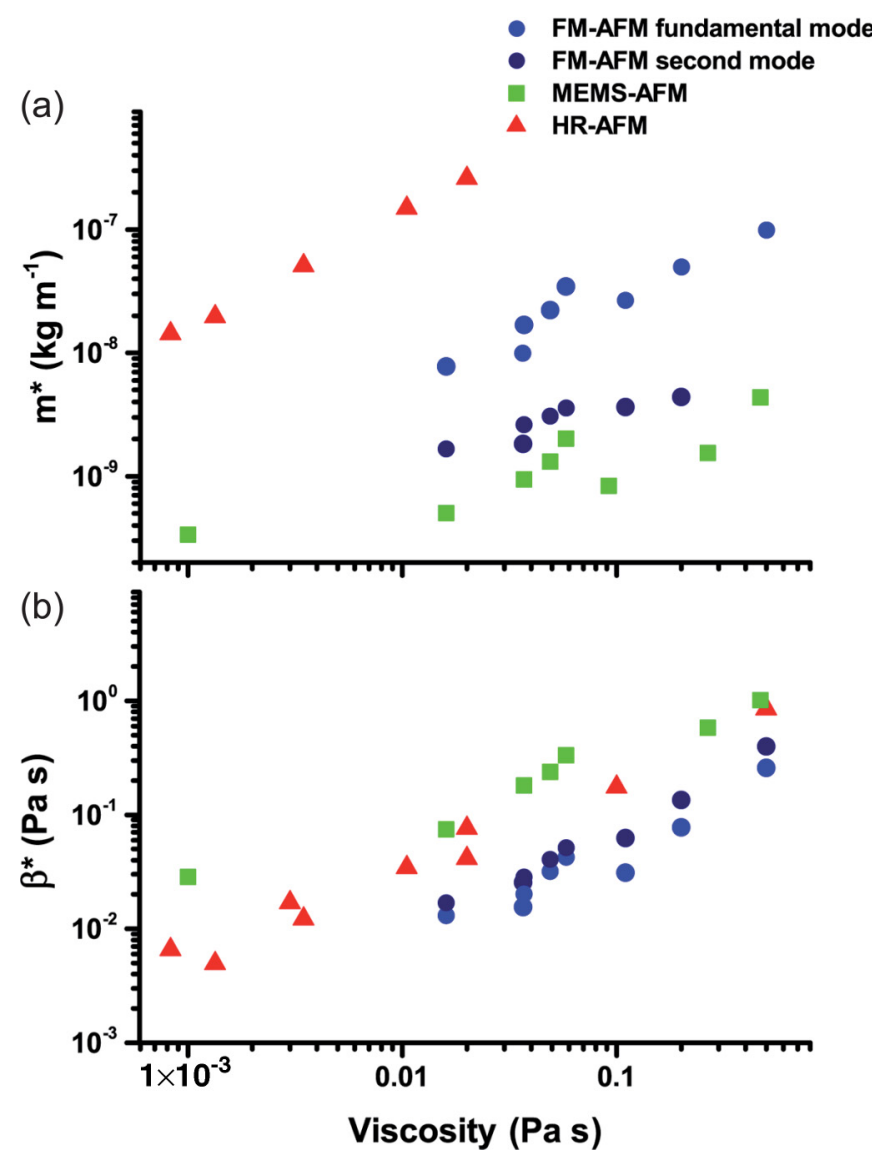

FIG. 5. (a) Added mass $m^{*}$ and (b) friction coefficient $\beta^{*}$ extracted from the three types of experiments, reported as a function of the liquid viscosity.

The added mass $m^{*}$ and friction coefficient $\beta^{*}$ were deduced from the FM AFM (for both fundamental and second modes), MEMS AFM, and HR AFM experiments on a large number of liquids. Examples of results are reported in Fig. 5. For clarity, we only reported several series of liquids. The whole set of measurements will be presented in Sec. V.

A general trend observed in Fig. 5 is that, for a given experiment, i.e., for fixed probe radius and excitation frequency, both quantities $m^{*}$ and $\beta^{*}$ increase with the liquid viscosity. The results also demonstrate that the values strongly depend on the type of AFM experiment. Since both probe radius $R$ and excitation angular frequency $\omega$ vary from experiment to experiment, their respective influences on the measurements are not straightforward. However, FM AFM can give an indication of the influence of frequency by comparing the response of the very same system (tip plus liquid) to excitations at the fundamental and second modes, which differs by a factor 6.25 in frequency. It appears that frequency has a small effect on the friction coefficient $\beta^{*}$, whereas the added mass term $m^{*}$ decreases by a factor of the order of 7 when increasing the frequency. This influence of excitation frequency may also explain the difference of three orders of magnitude between $m^{*}$ values obtained by MEMS AFM and HR AFM. On the contrary, these two techniques lead to similar $\beta^{*}$ values, significantly larger than the ones deduced from the FM AFM experiment. In order to interpret these results and give a unified vision of the whole data by disentangling the influence of the different parameters, we developed a model that is presented in the next section. 


\section{JULIEN DUPRÉ DE BAUBIGNY et al.}

\section{THEORETICAL MODEL}

In this section we establish the theoretical framework in which the hydrodynamic problem at stake can be understood. The hydrodynamics of rodlike particles has attracted a great deal of attention due to its implication in many important issues such as the rheological properties of solutions of such objects (polymers and nanotubes) or in size measurements from light scattering experiments $[2,4]$. Approximate solutions have been proposed for ellipsoids and cylinders of given aspect ratio totally immersed in an infinite liquid bath $[4,44]$. Since in our case the effects of the meniscus and of the immersed end may be considered as constant during the dipping process, we considered the case of an infinite cylinder oscillating longitudinally in an infinite liquid bath. The latter assumption is justified since in the experiments the diameter of the liquid vessel or droplet ( $6 \mathrm{~mm}$ for FM AFM and MEMS AFM and 14 mm for HR AFM) is much larger than the penetration depth of the shear waves in the liquids (of the order of 5-50 $\mu \mathrm{m}$ ). With that aim we revisited the model established by Batchelor for a cylinder moving steadily in a viscous liquid [45].

In order to compute the longitudinal drag force, we considered the flow induced by an infinite cylindrical rod oscillating along its axis in a purely viscous Newtonian liquid. The rod's radius is denoted by $R$ (see Fig. 1) and its speed, assumed to be harmonic (with no lack of generality due to the linearity of the equations), reads $v_{0} e^{-i \omega t}$. The velocity field $v(r, t)$ in the liquid is obtained by solving the cylindrical Stokes equation [43] $\varrho \partial_{t} v=\eta\left(\partial_{r}^{2} v+\frac{\partial_{r} v}{r}\right)$ together with a no-slip boundary condition at the rod's surface $v(R, t)=v_{0} e^{-i \omega t}$ and a vanishing-speed boundary condition at infinity $\lim _{r \rightarrow \infty} v(r, t)=0$. The solution reads, for all $r \geqslant R$,

$$
v(r, t)=v_{0} e^{-i \omega t} f\left(\frac{r}{\delta}, \frac{R}{\delta}\right),
$$

where $\delta=\sqrt{\frac{2 \eta}{\rho \omega}}$ is the skin thickness of the well-known two-dimensional (2D) case of a viscous flow induced by an oscillating plate in a liquid [43] and the function of two variables $f$ reads

$$
f\left(u_{1}, u_{2}\right)=\frac{\mathcal{K}_{0}\left[(1-i) u_{1}\right]}{\mathcal{K}_{0}\left[(1-i) u_{2}\right]},
$$

where $\mathcal{K}_{n}$ denotes the modified Bessel function of the second kind of order $n$ [46]. The real velocity field thus oscillates in an envelope $v_{e}(r)$ (see Fig. 6), whose derivation and expression are given in

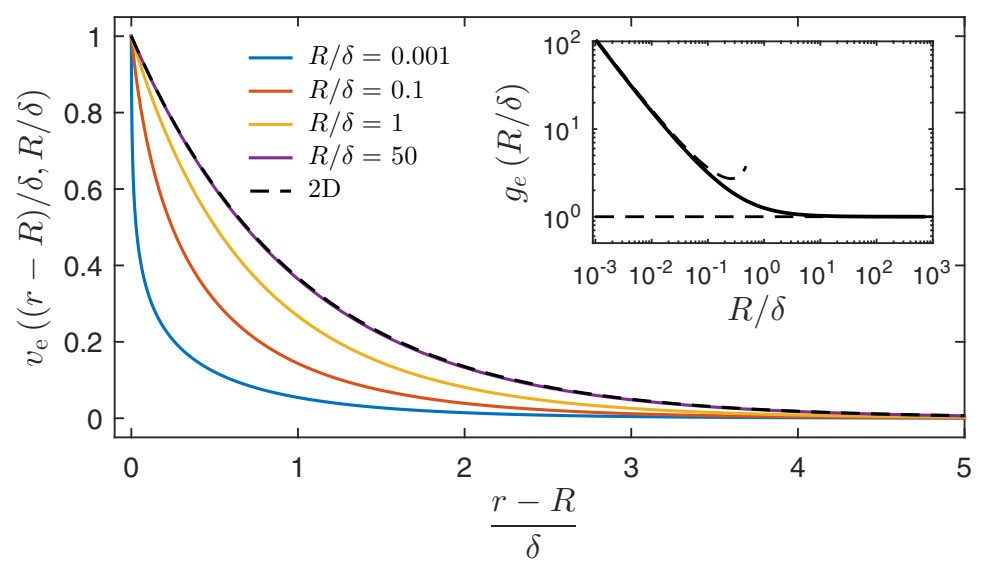

FIG. 6. Plot of the velocity envelope $v_{e}[(r-R) / \delta, \mathrm{R} / \delta]$ as a function of $(r-R) / \delta$ for different values of $R / \delta$. The black dashed curve corresponds to the well-known $2 \mathrm{D}$ viscous flow induced by an oscillating plane [43]. The inset shows a plot of $g_{e}\left(\frac{R}{\delta}\right)$, the envelope of the normalized shear stress $\mathrm{g}\left(\frac{R}{\delta}\right)$, given by Eq. (4). Dashed lines correspond to the asymptotic solutions for $R \gg \delta$ and $R \ll \delta$ [Eq. (5)]. 
[36]. When the radius $R$ is of order of or smaller than $\delta$, the velocity field becomes more confined than in the 2D solution leading to an effective skin smaller than $\delta$. This effective skin depth $\delta_{\text {eff }}$ will be evaluated quantitatively in the following. When $R \gg \delta$, the velocity profiles match that of the aforementioned $2 \mathrm{D}$ case. In the latter limit, the influence of the cylindrical geometry can thus be safely neglected.

The frictional force on the rod per unit area is given by the shear stress at the rod's surface

$$
\sigma=-\left.\sigma_{r z}\right|_{r=R}=-\left.\eta \partial_{r} v\right|_{r=R}=\frac{\eta}{\delta}(1-i) g\left(\frac{R}{\delta}\right) v_{0} e^{-i \omega t},
$$

where the function $g$ reads [46]

$$
g(u)=\frac{\mathcal{K}_{1}[(1-i) u]}{\mathcal{K}_{0}[(1-i) u]}=g_{1}(u)+i g_{2}(u),
$$

with $g_{1}$ and $g_{2}$ being, respectively, the real and imaginary parts of the function $g$. In Fig. 6 one can notice that as the radius $R$ becomes small compared to the 2D skin thickness $\delta$, the velocity gradient at the rod's surface increases dramatically. The shear stress oscillates with an amplitude $\sigma_{e}\left(\frac{R}{\delta}\right)=\sqrt{2} \frac{\eta}{\delta} v_{0} g_{e}\left(\frac{R}{\delta}\right)$. The stress enhancement factor $g_{e}\left(\frac{R}{\delta}\right)$ plotted in the inset of Fig. 6 (expression given in the Supplemental Material [36]) diverges for small $R / \delta$ as

$$
R / \delta \rightarrow 0, \quad g_{e}\left(\frac{R}{\delta}\right) \sim \frac{-1}{\sqrt{2} \frac{R}{\delta} \ln \left(\frac{R}{\delta}\right)} .
$$

In the case of the FM AFM experiments for which the ratio $R / \delta$ is of order $10^{-3}$, an enhancement of the stress by a factor 100 is found with respect to a planar situation. This leads to large shear rates of the order of $10^{5}-10^{6} \mathrm{~s}^{-1}$ in standard conditions.

Finally, the frictional force per unit length along the rod axis reads $F^{*}=2 \pi R \sigma$. Using Eq. (3), the force can be written in a temporal form as

$$
F^{*}=-\left.\beta^{*} v\right|_{r=R}-\left.m^{*} \partial_{t} v\right|_{r=R},
$$

where $\beta^{*}$ and $m^{*}$ are, respectively, a friction coefficient and a mass term, both per unit length. They read

$$
\beta^{*}=2 \pi \eta \frac{R}{\delta}\left(g_{1}(R / \delta)+g_{2}(R / \delta)\right)=2 \pi \eta C_{\beta}
$$

and

$$
m^{*}=2 \pi \frac{\eta}{\omega} \frac{R}{\delta}\left(g_{1}(R / \delta)-g_{2}(R / \delta)\right)=2 \pi \frac{\eta}{\omega} C_{m} .
$$

For high values of $R / \delta$ the friction coefficient and added mass are related through a simple expression, which is, as one might expect, that of the $2 \mathrm{D}$ situation

$$
R / \delta \rightarrow+\infty, \quad \beta^{*} \sim m^{*} \omega .
$$

The coefficients $C_{\beta}$ and $C_{m}$ are represented in Fig. 7 as a function of $R / \delta$. For large $R / \delta$ values, $C_{\beta}$ and $C_{m}$ meet the same asymptotic behavior $C_{\beta} \sim C_{m} \sim R / \delta$. The friction coefficient in this regime reads $\beta^{*} \sim m^{*} \omega \sim \pi R \sqrt{2 \eta \rho \omega}$. For small $R / \delta$, the asymptotic behavior of $C_{\beta}$ and $C_{m}$ reads

$$
R / \delta \rightarrow 0 \quad C_{\beta} \sim \frac{-1}{\ln \left(\frac{R}{\delta}\right)} \quad \text { and } \quad C_{m} \sim \frac{\pi / 4}{\left[\ln \left(\frac{R}{\delta}\right)\right]^{2}}
$$

In this regime, the coefficient $C_{\beta}$ is weakly dependent on the probe size, liquid viscosity $\eta$, and excitation frequency $\omega$ leading to $\beta^{*} \sim-2 \pi \eta / \ln (R / \delta)$. The mass term reads $m^{*} \sim \frac{\pi^{2}}{2} \frac{\eta}{\omega} \frac{1}{[\ln (R / \delta)]^{2}}$.

We may now use the previous results to evaluate the aforementioned effective skin depth $\delta_{\text {eff }}$, by analogy with the $2 \mathrm{D}$ situation. In the latter case indeed, the added mass is half the mass of the 


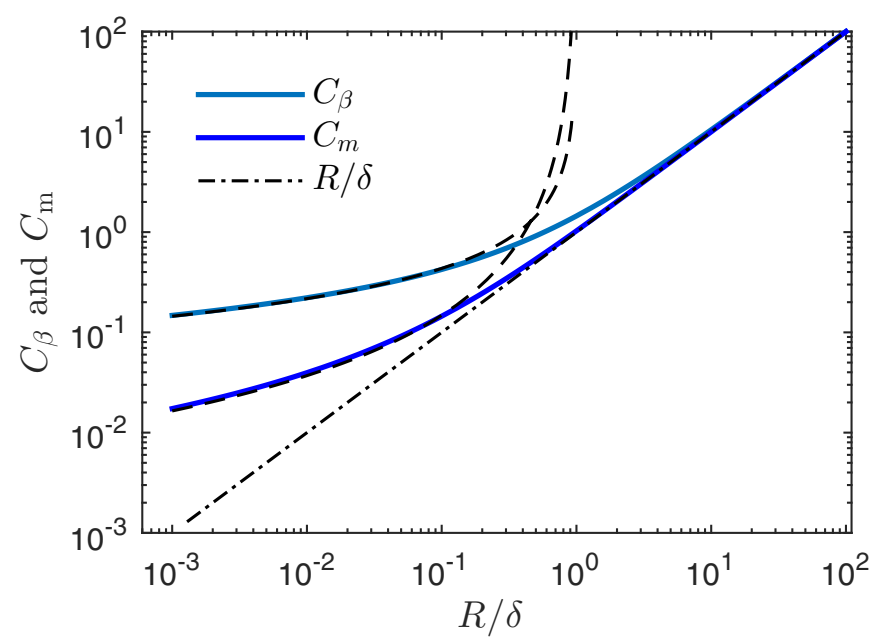

FIG. 7. Plot of $C_{\beta}$ and $C_{m}$ [see Eqs. (7) and (8)] as a function of $R / \delta$ (solid curves). The dashed black curves display the asymptotic regime as given by Eq. (10) and the dash-dotted black curve displays the $2 \mathrm{D}$ regime.

fluid localized in the viscous layer of depth $\delta$. We take therefore $\delta_{\text {eff }}$ such that the mass per unit length of an annular section of liquid with inner radius $R$ and outer radius $R+\delta_{\text {eff }}$ is equal to $2 m^{*}$. Identifying with Eq. (8), one has

$$
\frac{\delta_{\text {eff }}}{\delta}=-\frac{R}{\delta}+\sqrt{\left(\frac{R}{\delta}\right)^{2}+2 C_{m}} .
$$

Figure 8 represents the ratio $\delta_{\text {eff }} / \delta$ as a function of $R / \delta$. For large values of $R / \delta$, one recovers, as expected, the 2D regime for which $\delta_{\text {eff }}=\delta$ (dashed red curve). When $R$ is of order of or smaller than $\delta$, the effective skin length $\delta_{\text {eff }}$ decreases and reaches the small radius asymptotic regime (dashed

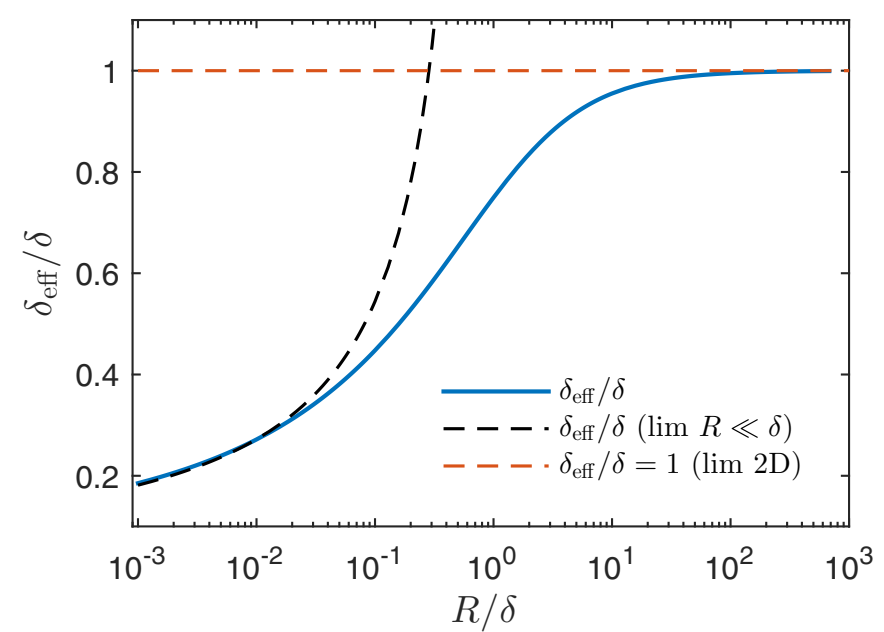

FIG. 8. Plot of $\delta_{\text {eff }} / \delta$ as given by Eq. (11) as a function of $R / \delta$ (solid blue curve). The dashed red line displays the $2 \mathrm{D}$ regime for which $\delta_{\text {eff }}=\delta$ and the dashed black curve displays the small radius asymptotic regime as given by Eq. (12). 


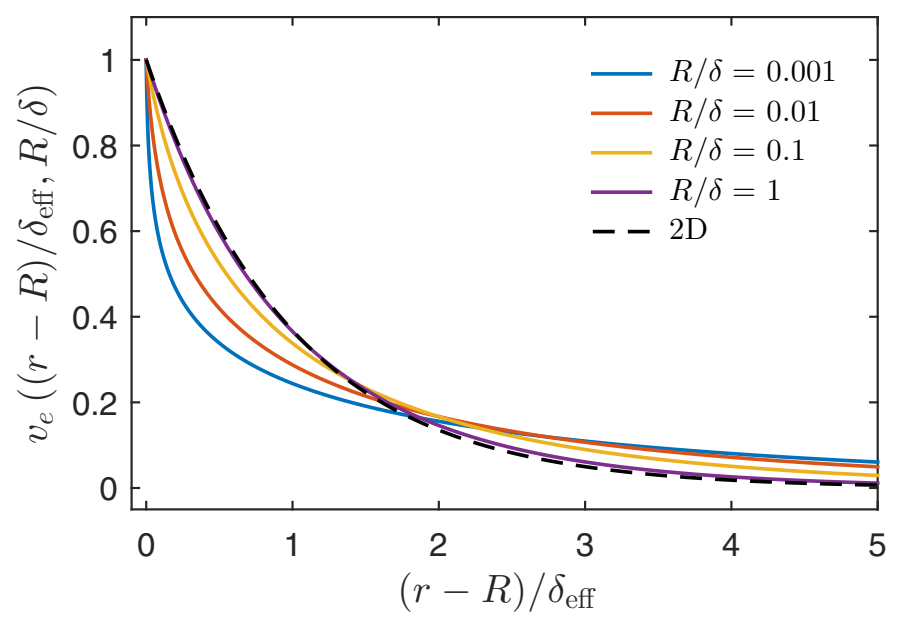

FIG. 9. Plot of the velocity envelope $v_{\mathrm{e}}\left[(r-R) / \delta_{\text {eff }}, \mathrm{R} / \delta\right]$ as a function of the rescaled variable $(r-R) / \delta_{\text {eff }}$ for different values of $R / \delta$. The black dashed curve corresponds to the $2 \mathrm{D}$ viscous flow induced by an oscillating plane.

black curve), for which $R$ can be neglected in the calculation of the annular section area, leading to

$$
R / \delta \rightarrow 0, \quad \frac{\delta_{\text {eff }}}{\delta} \sim \sqrt{2 C_{m}} \sim \frac{-\sqrt{\pi / 2}}{\ln \left(\frac{R}{\delta}\right)}
$$

Equation (11) gives a quantitative description of the extension of the velocity field around a nanocylinder. For probes with cylinder radius much smaller than the thickness of the $2 \mathrm{D}$ viscous layer $\delta$, the extension of the viscous layer is significantly reduced. One observes that $\delta_{\text {eff }} / \delta$ decreases dramatically when $R / \delta$ decreases from 10 to $10^{-2}$ (with a drop of approximately $80 \%$ ). A further decrease of the probe size has a small (logarithmic) effect.

Contrary to the friction coefficient $\beta^{*}$, which is only sensitive to the shear stress at the solid-liquid boundary, the added mass takes into account the whole velocity profile. In particular, in the limit $R / \delta \rightarrow 0$ the friction coefficient decreases less strongly than the added mass. This can be understood using a simple argument. Figure 9 displays the velocity profiles as a function of the rescaled variable $(r-R) / \delta_{\text {eff }}$. In this representation, the rescaled added mass does not vary with $R / \delta$, while the rescaled shear stress at the solid-liquid boundary still significantly increases in amplitude as $R / \delta$ is decreased. This interesting feature that explains the difference between $C_{\beta}$ and $C_{m}$ for $R / \delta \ll 1$ is inherent to the cylindrical geometry of the system. When $R / \delta>1$ the normalized curves of Fig. 9 are superposed and one regains a quasi-2D geometry and recovers the well-known relation $\beta=m \omega$.

\section{DISCUSSION}

The model described above provides a comprehensive description of the behavior of the viscous layer as a function of the experimental conditions. It was shown that different regimes may occur as a function of the relative values of the probe size $R$ and the relevant length scale $\delta$ of the problem, which depends on the liquid properties $\eta$ and $\rho$ and the excitation angular frequency $\omega$ through $\delta=\sqrt{\frac{2 \eta}{\rho \omega}}$. Typical values of $R$ and $\delta$ are reported in Table I. It shows that the small probe diameters used in FM AFM, significantly smaller than the thickness $\delta$, provide a way to assess the regime of small $\frac{R}{\delta}\left(\frac{R}{\delta} \sim 6 \times 10^{-4}-2 \times 10^{-2}\right)$ where geometrical aspects are important. In MEMS AFM, the high frequencies lead to small submicrometric $\delta$ values that may be comparable to the probe size. 


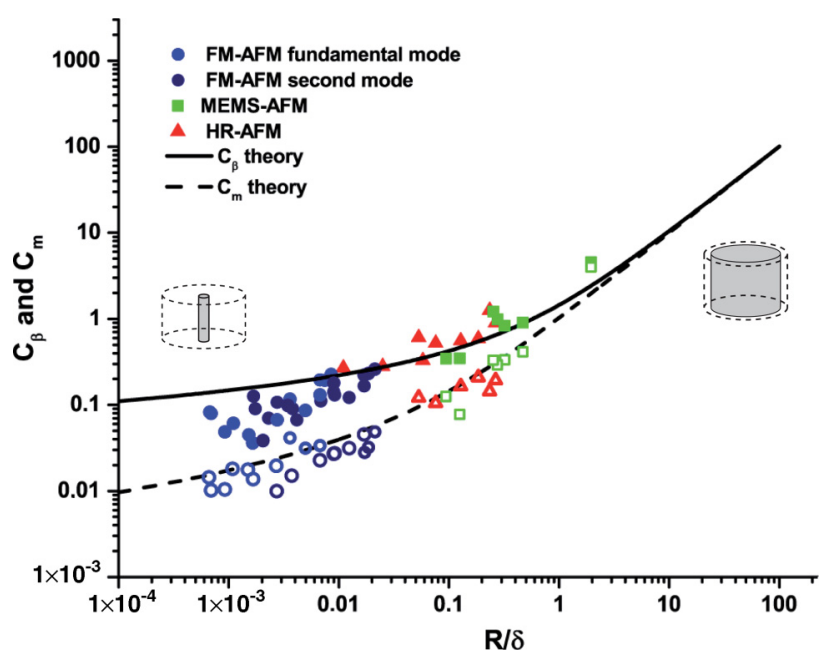

FIG. 10. Master curve of the $C$ coefficient determined as $C_{\beta}=\frac{\beta^{*}}{2 \pi \eta}$ (closed symbols) or $C_{m}=\frac{m^{*} \omega}{2 \pi \eta}$ (open symbols) as a function of $R / \delta$ for the whole set of experiments with the FM AFM (fundamental mode in light blue circles, second mode in dark blue circles), MEMS AFM (green squares), and HR AFM (red triangles). The solid line corresponds to the theoretical value values of $C_{\beta}$ expressed in Eq. (7), the dashed line to the one of $C_{m}$ expressed in Eq. (8).

HR AFM uses large probes whose radius is also of the same order as $\delta$. These last cases therefore allow one to access an intermediate regime approaching the $2 \mathrm{D}$ case $\left(\frac{R}{\delta} \sim 1 \times 10^{-2}-1\right)$.

The fact that MEMS AFM and HR AFM give access to the same range of $R / \delta$ values explains that these techniques lead to similar values of the friction coefficient $\beta^{*}$. Since $C_{\beta}$ is an increasing function of $R / \delta$, these values are also expected to be larger than the ones from FM AFM as observed in Fig. 5. The same trends are expected for $m^{*} \omega$, which is consistent with the strong influence of the excitation frequency on the added mass term discussed in Sec. III.

In order to assess more quantitatively the model, the whole set of experimental results was compared to the model using the master curves defined by Eqs. (7) and (8) and represented in Fig. 10. In this representation, we plotted for each experimental data point the value of the coefficient $C_{\beta}$ and $C_{m}$ defined as $C_{\beta}=\frac{\beta^{*}}{2 \pi \eta}$ and $C_{m}=\frac{m^{*} \omega}{2 \pi \eta}$ as a function of the ratio $R / \delta$. Large values of $R / \delta$ correspond to the $2 \mathrm{D}$ situation, whereas the cylindrical geometry needs to be taken into account for $\frac{R}{\delta} \ll 1$, as sketched in the insets of Fig. 10. Interestingly, the large range of probe sizes, solicitation conditions, and liquid viscosities provided by the combination of all AFM techniques allows us to vary the $R / \delta$ parameter over more than three orders of magnitude, which corresponds to six orders of magnitude in Reynolds number.

The whole set of experimental data points is reported in Fig. 10, where closed symbols and the solid line correspond to experimental and theoretical values of the dissipation $C_{\beta}$ parameter, whereas open symbols and the dashed line represent the mass $C_{m}$ parameter. The colors are associated with the measurement techniques, namely, blue (dark blue) for the fundamental (second) mode in FM $\mathrm{AFM}$, red for HR AFM, and green for MEMS AFM. We observe that each series of points $\left(C_{\beta}\right.$ and $C_{m}$ ) gathers well on a single curve, demonstrating the pertinence of the $R / \delta$ parameter to describe the liquid behavior. All data also show good agreement with the model. More precisely, the mass term is very well reproduced by the model. This is also the case for the dissipation data, except for the points corresponding to $R / \delta<5 \times 10^{-3}$, which were obtained by FM AFM on the most viscous liquids. In this case, the experimental data are overestimated by about $30 \%-50 \%$ by the model. The origin of this discrepancy remains unclear. No particular issue can be anticipated from the measurement side. Some assumptions such as the no-slip boundary condition could potentially be invalidated at the nanoscale. The calculations can be revised with a Navier-like slip boundary 
condition, which has the effect of reducing the shear stress and thus reducing the friction coefficient. While for simple liquids on hydrophilic surfaces it is commonly assumed that slippage can be safely neglected [16], interestingly, the points that correspond to low- $R / \delta$ values were obtained with ionic liquids, which exhibit a strong structuration at the solid surface as demonstrated by surface force apparatus [35]. The strong stress enhancement due to geometrical aspects discussed in the previous section may induce a slip in this particular case.

It is worth noting that MEMS AFM is based on a purely vertical motion of the tip and therefore avoids any sideways motion of the fiber inherent to cantilever based oscillation. The latter effect, which depends on the ratio of the fiber to cantilever lengths, can be safely neglected for FM AFM but can lead to overestimation of the $C_{\beta}$ coefficient up to $20 \%$ for HR AFM, which remains within the uncertainty of the measurement (see the Supplemental Material [36]).

Since no adjustable parameter is present in the theory, this study demonstrates that the experimental protocol together with the methods used to interpret the data and obtain physical parameters such as added mass and friction coefficient is robust. It establishes that FM AFM, MEMS AFM, and HR AFM are techniques that can be used with confidence for quantitative investigation of dissipation processes at micro- and nanoscales.

According to the description in terms of the effective thickness of the viscous layer discussed above [Eq. (11) and Fig. 8], the experiments performed in FM AFM mode correspond to values of the effective thickness of the order of $\delta_{\text {eff }} \sim 0.2 \delta$, whereas for MEMS AFM and HR AFM the dynamic confinement is moderate with $\delta_{\text {eff }} \sim 0.5 \delta-0.8 \delta$. This quantifies the intuitive fact that, for a small tip radius, the velocity field extends less in the liquid than for the $2 \mathrm{D}$ situation of an oscillating plane. Even if the reduction of the probe dimension limits the extension of the velocity field compared to the 2D situation, this reduction remains rather small even for a tip radius 1000 times smaller than the thickness of the viscous layer. The fact that the velocity field extends over several microns may have important implications for the collective motion of passive or active nanoparticles in a liquid environment. The effective thicknesses of the viscous layer are therefore of the order of $\delta_{\text {eff }} \sim 1-10 \mu \mathrm{m}, \delta_{\text {eff }} \sim 0.1-1 \mu \mathrm{m}$, and $\delta_{\text {eff }} \sim 5-50 \mu \mathrm{m}$ for FM AFM, MEMS AFM, and HR AFM, respectively.

\section{CONCLUSION}

In this article we have shown that AFM techniques, combined with a model tip geometry, are powerful tools for the quantitative study of hydrodynamics in a viscous layer around a micro- or nanocylindrical probe. We implemented three different methods and defined protocols allowing the independent measurement of the added mass and friction terms over a large range of probe size, fluid viscosity, and excitation frequency. A model was developed to account for the experimental observations. It shows that the relevant parameter is the ratio $R / \delta$ of the probe size $R$ to the 2D viscous layer thickness $\delta$. All experimental data can be gathered on two master curves, one for added mass and one for the friction coefficient, using the $R / \delta$ ratio as the single control parameter spanning over three orders of magnitude. They are quantitatively reproduced by the theoretical model without any adjustable parameter, showing the potential of AFM to measure dissipation processes in liquids down to the nanometer scale.

The theoretical model allows quantifying the extension of the velocity field around the nanoprobe and shows that it is significantly lower than the thickness of a $2 \mathrm{D}$ viscous layer $\delta$. This confinement is associated with a strong enhancement of the surface stress as the probe size is decreased. The scaling laws provided by the model are of interest for the development of nanorheology, in particular for complex fluids that may exhibit nano- to microscale characteristic lengths. In this context, the MEMS AFM experiment allows probing small liquid volumes with characteristic length in the $100 \mathrm{~nm}$ range and is also relevant in the recent field of high-frequency nanofluidics $[23,31,47]$.

The quantitative measurement of dissipation processes in liquid around a nanometer scale probe provided by this study may also open the way for systematic investigation of dissipation 


\section{JULIEN DUPRÉ DE BAUBIGNY et al.}

in intrinsically small liquid volumes such as nanomenisci or at contact lines, issues that are not fully understood despite their great importance in wetting science [48].

\section{ACKNOWLEDGMENTS}

We thank Elie Raphaël and Sergio Ciliberto for fruitful discussions, Philippe Demont for his help in the measurements of ionic liquid viscosity, and Christopher Madec for contribution to the HR AFM experiment. This study was partially supported through the ANR by the NANOFLUIDYN project (Grant No. ANR-13-BS10-0009) and Laboratory of Excellence NEXT (Grant No. ANR-10LABX-0037) in the framework of the "Programme des Investissements d'Avenir." Financial support from the ERC project OutEFLUCOP (Grant No. 267687) is also acknowledged.

[1] Nanoscale Liquid Interfaces: Wetting, Patterning and Force Microscopy at the Molecular Scale, edited by T. Ondarcuhu and J. P. Aime (Pan Stanford Publishing, Singapore, 2013).

[2] M. A. Tracy and R. Pecora, Dynamics of rigid and semirigid rodlike polymers, Ann. Rev. Phys. Chem. 43, 525 (1992).

[3] J. J. Stickel and R. L. Powell, Fluid mechanics and rheology of dense suspensions, Annu. Rev. Fluid Mech. 37, 129 (2005).

[4] M. L. Mansfield and J. F. Douglas, Transport properties of rodlike particles, Macromolecules 41, 5422 (2008).

[5] D. A. Sessoms, A. Amon, L. Courbin, and P. Panizza, Complex Dynamics of Droplet Traffic in a Bifurcating Microfluidic Channel: Periodicity, Multistability, and Selection Rules, Phys. Rev. Lett. 105, 154501 (2010).

[6] J. Bleibel, A. Dominguez, F. Gunther, J. Harting, and M. Oettel, Hydrodynamic interactions induce anomalous diffusion under partial confinement, Soft Matter 10, 2945 (2014).

[7] D. M. Wootton and D. N. Ku, Fluid mechanics of vascular systems, diseases, and thrombosis, Annu. Rev. Biomed. Eng. 1, 299 (1999).

[8] S. Gravelle, L. Joly, F. Detcheverry, C. Ybert, C. Cottin-Bizonne, and L. Bocquet, Optimizing water permeability through the hourglass shape of aquaporins, Proc. Natl. Acad. Sci. USA 110, 16367 (2013).

[9] A. Bricard, J.-B. Caussin, N. Desreumaux, O. Dauchot, and D. Bartolo, Emergence of macroscopic directed motion in populations of motile colloids, Nature (London) 503, 95 (2013).

[10] I. Theurkauff, C. Cottin-Bizonne, J. Palacci, C. Ybert, and L. Bocquet, Dynamic Clustering in Active Colloidal Suspensions with Chemical Signaling, Phys. Rev. Lett. 108, 268303 (2012).

[11] A. Zoettl and H. Stark, Hydrodynamics Determines Collective Motion and Phase Behavior of Active Colloids in Quasi-Two-Dimensional Confinement, Phys. Rev. Lett. 112, 118101 (2014).

[12] G. Loget and A. Kuhn, Propulsion of microobjects by dynamic bipolar self-regeneration, J. Am. Chem. Soc. 132, 15918 (2010).

[13] J. Vermant and M. J. Solomon, Flow-induced structure in colloidal suspensions, J. Phys.: Condens. Matter 17, R187 (2005).

[14] F. Jin and C. Wu, Observation of the First-Order Transition in Ultrafiltration of Flexible Linear Polymer Chains, Phys. Rev. Lett. 96, 237801 (2006).

[15] A. Lucas, C. Zakri, M. Maugey, M. Pasquali, P. van der Schoot, and P. Poulin, Kinetics of nanotube and microfiber scission under sonication, J. Phys. Chem. C 113, 20599 (2009).

[16] L. Bocquet and E. Charlaix, Nanofluidics, from bulk to interfaces, Chem. Soc. Rev. 39, 1073 (2010).

[17] T. M. Squires and T. G. Mason, Fluid mechanics of microrheology, Annu. Rev. Fluid Mech. 42, 413 (2010).

[18] T. A. Waigh, Microrheology of complex fluids, Rep. Prog. Phys. 68, 685 (2005).

[19] M. Atakhorrami, D. Mizuno, G. H. Koenderink, T. B. Liverpool, F. C. MacKintosh, and C. F. Schmidt, Short-time inertial response of viscoelastic fluids measured with Brownian motion and with active probes, Phys. Rev. E 77, 061508 (2008).

[20] E. Roeben, L. Roeder, S. Teusch, M. Effertz, U. K. Deiters, and A. M. Schmidt, Magnetic particle nanorheology, Colloid Polymer Sci. 292, 2013 (2014). 
[21] B. Jakoby, R. Beigelbeck, F. Keplinger, F. Lucklum, A. Niedermayer, E. K. Reichel, C. Riesch, T. Voglhuber-Brunnmaier, and B. Weiss, Miniaturized sensors for the viscosity and density of liquidsPerformance and issues, IEEE Trans. Ultrason. Ferroelectr. Freq. Control 57, 111 (2010).

[22] J. J. Crassous, R. Regisser, M. Ballauff, and N. Willenbacher, Characterization of the viscoelastic behavior of complex fluids using the piezoelastic axial vibrator, J. Rheol. 49, 851 (2005).

[23] D. M. Karabacak, V. Yakhot, and K. L. Ekinci, High-Frequency Nanofluidics: An Experimental Study using Nanomechanical Resonators, Phys. Rev. Lett. 98, 254505 (2007).

[24] I. Lee, K. Park, and J. Lee, Precision viscosity measurement using suspended microchannel resonators, Rev. Sci. Instrum. 83, 116106 (2012).

[25] H. L. Ma, J. Jimenez, and R. Rajagopalan, Brownian fluctuation spectroscopy using atomic force microscopes, Langmuir 16, 2254 (2000).

[26] A. Roters, M. Schimmel, J. Ruhe, and D. Johannsmann, Collapse of a polymer brush in a poor solvent probed by noise analysis of a scanning force microscope cantilever, Langmuir 14, 3999 (1998).

[27] C. Devailly, J. Laurent, A. Steinberger, L. Bellon, and S. Ciliberto, Mode coupling in a hanging-fiber AFM used as a rheological probe, Europhys. Lett. 106, 54005 (2014).

[28] X. M. Xiong, S. O. Guo, Z. L. Xu, P. Sheng, and P. Tong, Development of an atomic-force-microscopebased hanging-fiber rheometer for interfacial microrheology, Phys. Rev. E 80, 061604 (2009).

[29] C. Jai, J. P. Aime, D. Mariolle, R. Boisgard, and F. Bertin, Wetting an oscillating nanoneedle to image an air-liquid interface at the nanometer scale: Dynamical behavior of a nanomeniscus, Nano Lett. 6, 2554 (2006).

[30] D. Johannsmann, Viscoelastic, mechanical, and dielectric measurements on complex samples with the quartz crystal microbalance, Phys. Chem. Chem. Phys. 10, 4516 (2008).

[31] M. Pelton, D. Chakraborty, E. Malachosky, P. Guyot-Sionnest, and J. E. Sader, Viscoelastic Flows in Simple Liquids Generated by Vibrating Nanostructures, Phys. Rev. Lett. 111, 244502 (2013).

[32] B. Legrand, D. Ducatteau, D. Theron, B. Walter, and H. Tanbakuchi, Detecting response of microelectromechanical resonators by microwave reflectometry, Appl. Phys. Lett. 103, 053124 (2013).

[33] B. Walter, M. Faucher, E. Algre, B. Legrand, R. Boisgard, J. P. Aime, and L. Buchaillot, Design and operation of a silicon ring resonator for force sensing applications above $1 \mathrm{MHz}, \mathrm{J}$. Micromech. Microeng. 19, 115009 (2009).

[34] P. Paolino, F. A. A. Sandoval, and L. Bellon, Quadrature phase interferometer for high resolution force spectroscopy, Rev. Sci. Instrum. 84, 095001 (2013).

[35] S. Perkin, Ionic liquids in confined geometries, Phys. Chem. Chem. Phys. 14, 5052 (2012).

[36] See Supplemental Material at http://link.aps.org/supplemental/10.1103/PhysRevFluids.1.044104 for chemical structure and characteristic properties of the liquids used, MEMS AFM description, HR AFM on viscous liquids, effect of oscillation amplitude on FM AFM and MEMS AFM, velocity profile and shear stress, and sideway motion of the fiber on an AFM cantilever.

[37] H. J. Butt and M. Jaschke, Calculation of thermal noise in atomic-force microscopy, Nanotechnology $\mathbf{6}$, 1 (1995).

[38] A. H. Barber, S. R. Cohen, and H. D. Wagner, Static and Dynamic Wetting Measurements of Single Carbon Nanotubes, Phys. Rev. Lett. 92, 186103 (2004).

[39] M. Delmas, M. Monthioux, and T. Ondarçuhu, Contact Angle Hysteresis at the Nanometer Scale, Phys. Rev. Lett. 106, 136102 (2011).

[40] M. M. Yazdanpanah, M. Hosseini, S. Pabba, S. M. Berry, V. V. Dobrokhotov, A. Safir, R. S. Keynton, and R. W. Cohn, Micro-Wilhelmy and related liquid property measurements using constant-diameter nanoneedle-tipped atomic force microscope probes, Langmuir 24, 13753 (2008).

[41] J. Dupré de Baubigny, M. Benzaquen, L. Fabie, M. Delmas, J.-P. Aime, M. Legros, and T. Ondarcuhu, Shape and effective spring constant of liquid interfaces probed at the nanometer scale: Finite size effects, Langmuir 31, 9790 (2015).

[42] F. J. Giessibl, Advances in atomic force microscopy, Rev. Mod. Phys. 75, 949 (2003).

[43] L. D. Landau and E. M. Lifshitz, Course of Theoretical Physics: Fluid Mechanics (Elsevier, Oxford, 1987).

[44] S. Broersma, Viscous force constant for a closed cylinder, J. Chem. Phys. 32, 1632 (1960). 


\section{JULIEN DUPRÉ DE BAUBIGNY et al.}

[45] G. K. Batchelor, The skin friction on infinite cylinders moving parallel to their length, Q. J. Mech. Appl. Math. 7, 179 (1954).

[46] Handbook of Mathematical Functions: With Formulas, Graphs, and Mathematical Tables, edited by M. Abramowitz and I. A. Stegun (Courier Dover, New York, 1972).

[47] S. Kheifets, A. Simha, K. Melin, T. Li, and M. G. Raizen, Observation of Brownian motion in liquids at short times: Instantaneous velocity and memory loss, Science 343, 1493 (2014).

[48] J. H. Snoeijer and B. Andreotti, Moving contact lines: Scales, regimes, and dynamical transitions, Ann. Rev. Fluid. Mech. 45, 269 (2013). 